\title{
Multiple embolic strokes in a young woman
}

\author{
Authors: Amr Kandil, ${ }^{A}$ Rajinder Bilku, ${ }^{B}$ Pancras Felix ${ }^{B}$ and Rajah Nata ${ }^{B}$
}

\section{Introduction}

The list of the possible stroke aetiologies among young adults is extensive. Cerebral vascular abnormality and cardiac embolic causes are the main differentials, with the latter accounting for one-third of strokes in young adults. We present a young woman with multiple embolic strokes.

\section{Case presentation}

Our patient was a 44-year-old woman. She was known to have hypertension and chronic kidney disease. She had also been admitted to our hospital 1 year earlier with chest pain, left bundle branch block (LBBB), and minimally elevated troponin and had no significant coronary artery disease on her coronary angiography. On the most recent admission, she presented with headache and dizziness. She was hypertensive with no fever, no neck rigidity or photophobia and with normal neurological and other systemic examination. Computed tomography (CT) of the brain was done followed by lumbar puncture, and both were clear. Brain magnetic resonance imaging (MRI) showed multiple small infarctions on both cerebral hemispheres, with a clinical impression of likely multiple embolic strokes.

An echocardiography showed left ventricular hypertrophy $(\mathrm{LVH})$, normal systolic function, thickening of both mitral valve leaflets with a small mobile mass attached to the anterior mitral valve leaflet. A transoesophageal echocardiography confirmed a small echo-dense mobile mass attached to the anterior mitral valve leaflet, as well as an accidentally discovered right atrial mass attached to the interatrial septum of unknown aetiology with no evidence of patent foramen ovale.

Urgent cardiac MRI showed a $13 \times 10 \mathrm{~mm}$ mass within the inferior aspect of the right atrium with no late gadolinium enhancement (LGE), raising the possibility of a thrombus; however, myxoma could not be ruled out. It showed also a $7 \times 4 \mathrm{~mm}$ mass along the atrial aspect of the anterior mitral leaflet with LGE (Fig 1). This could not be assessed due to its small size. There was LVH with the LV mildly dilated with preserved systolic function. It showed also mild LGE within the basal inferolateral wall, suggesting possible myocarditis.

Looking at all the results, there was no clear pathological correlation between both masses and the clinical impression

Authors: ${ }^{A}$ Norfolk and Norwich University Hospital, Norwich, UK; ${ }^{B}$ Queen Elizabeth Hospital, King's Lynn, UK was embolic strokes related to the left side mitral mass and an accidentally discovered right atrial mass, with a possibility of right atrial myxoma as a dual pathology. An urgent referral for surgical removal of the masses in the right atrium and from the mitral valve was then made. Histopathological examination revealed the right atrial mass was an organising thrombus and the mitral valve mass showed neutrophilic collection, fibrin, fibro-elastic fragments and a picture suggestive of inflammatory scarring and no organisms were detected.

Our patient had chronic kidney disease of no clear aetiology, multiple embolic strokes with a mitral valve mass, right atrial thrombus and evidence of focal myocarditis. A provisional working diagnosis of systemic lupus erythematosus (SLE) was made with sterile mitral valve vegetation (Libman-Sacks endocarditis). Her antinuclear antibodies (ANA) and dsDNA were negative and her Lupus anticoagulant was positive on one occasion. She was started on oral anticoagulation and was referred for renal biopsy to confirm the diagnosis, but the patient did not attend follow-up.
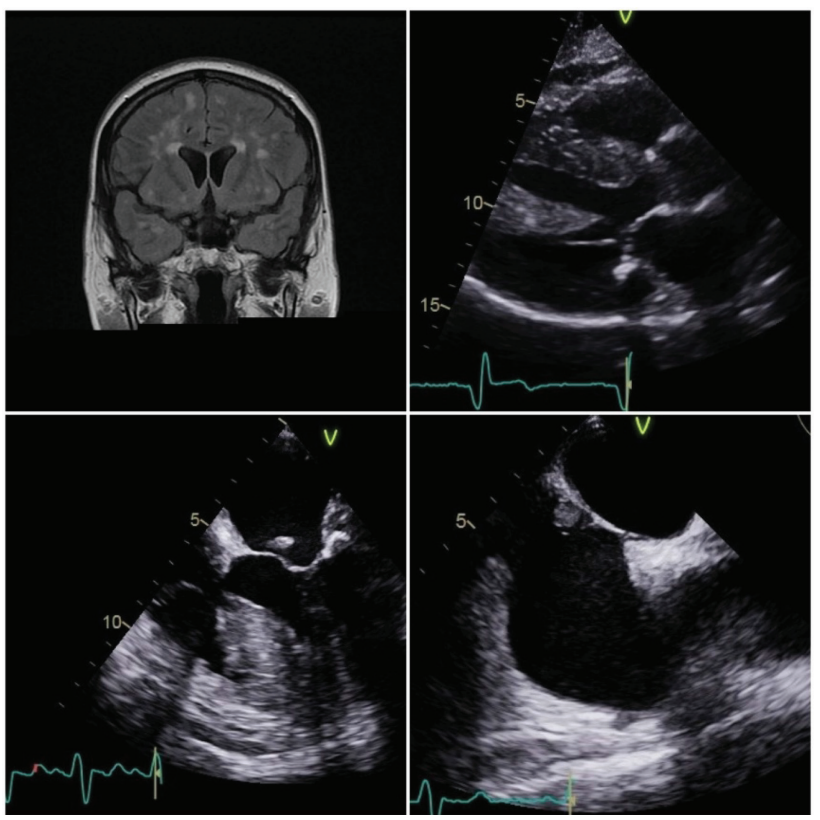

Fig 1. Top left: brain magnetic resonance imaging showing multiple bilateral embolic strokes. Top right: left ventricular hypertrophy with hypertrophied papillary muscles. Bottom left: anterior mitral leaflet mass. Bottom right: right atrial mass attached to the interatrial septum. 


\section{Discussion}

ANA-negative SLE has been infrequently described in the literature over the past 30 years. One of the causes of negative ANA in SLE patients is persistent proteinuria with renal loss of immunoglobulins, as in our case.

Our patient had an open-heart surgery which was probably unnecessary given the position of the mass, which is atypical of myxoma, and it could be postponed pending full investigation and probably a trial of anticoagulation. However, as in most right atrial masses, there are usually diagnostic confusions (and surgical approach appeared to be the safest strategy).

\section{Conflicts of interest}

None declared. 